\title{
Loss of connectivity in Alzheimer's disease: an evaluation of white matter tract integrity with colour coded MR diffusion tensor imaging
} Stephen E Rose, Fang Chen, Jonathan B Chalk, Fernando O Zelaya, Wendy E Strugnell,
Mark Benson, James Semple, David M Doddrell

\begin{abstract}
A novel MRI method-diffusion tensor imaging-was used to compare the integrity of several white matter fibre tracts in patients with probable Alzheimer's disease. Relative to normal controls, patients with probable Alzheimer's disease showed a highly significant reduction in the integrity of the association white matter fibre tracts, such as the splenium of the corpus callosum, superior longitudinal fasciculus, and cingulum. By contrast, pyramidal tract integrity seemed unchanged. This novel finding is consistent with the clinical presentation of probable Alzheimer's disease, in which global cognitive decline is a more prominent feature than motor disturbance.

(F Neurol Neurosurg Psychiatry 2000;69:528-530)
\end{abstract}

Keywords: Alzheimer's disease; diffusion tensor MRI

Although Alzheimer's disease is generally considered to affect grey matter, histological studies show pathological changes, such as a loss of axons and oligodendrocytes together with a reactive astrocytosis in the white matter. ${ }^{1}$ Magnetic resonance diffusion tensor imaging (DTI) is a non-invasive technique that can identify white matter tracts (WMTs) by evaluating the bulk diffusion of water in three dimensions; WMTs appear relatively brighter compared with surrounding tissues on DTI because the method identifies regions that are anisotropic, or areas where the diffusion of water is significantly greater in one direction than in others. The lattice index $(\mathrm{LI})^{2}$ can be used as a quantitative measure of anisotropy in DTI. In addition, the direction of the bulk water diffusion can be determined and the orientation of WMTs, such as the corpus callosum, depicted by appropriate colour coding. ${ }^{3}$ When WMTs degenerate, as occurs in cerebral infarction, there is a decrease in anisotropy and a corresponding reduction in the LI. ${ }^{4}$ Colour coded anisotropy maps have also been successfully used to study the structural integrity of pyramidal tract fibres in traumatic injury of the internal capsule. ${ }^{5}$ Hence, DTI may give new insights into the pathology of neurological conditions such as Alzheimer's disease by providing information on the structural integrity, or LI, of WMTs. Patients with early to moderate probable Alzheimer's disease have significantly impaired cognition but have slow-normal mobility. ${ }^{6}$ In this study we hypothesised that, when compared with aged matched normal controls, DTI would show significant reductions in the LI of the association fibre WMTs compared with the LI of the pyramidal tracts.

\section{Methods}

Patients referred for investigation of dementia were evaluated with a history, an examination, and a neuropsychological assessment and excluded if there was evidence of head trauma, a primary psychiatric diagnosis, stroke, hydrocephalus, or a metabolic, infectious, or endocrine cause of dementia. Patients fulfilling the criteria for frontotemporal dementia were excluded, ${ }^{7}$ as were patients with pyramidal or extrapyramidal tract signs. Patients were excluded if T2 weighted MRI showed deep white matter hyperintensites. Nine healthy older adults (aged 66-80, mean 72 (SD 0.7) years, mini mental state examination $(\mathrm{MMSE})^{8}>28$ ) were control subjects. Eleven patients (aged 49-80, mean 70 (SD 0.6) years, MMSE 6-21) who had a diagnosis of probable Alzheimer's disease, as defined by the National Institute of Neurological and Communicative Disorders and Stroke-Alzheimer's Disease and Related Disorders Association (NINCDS) ${ }^{9}$ and dementia diagnosed from the diagnostic and statistical manual of mental disorders, 3rd ed, revised criteria for Alzheimer's disease, ${ }^{10}$ took part in the study. Patients with probable Alzheimer's disease and controls were reexamined at 3 to 6 month intervals by clinical examination and with the MMSE. Patients with probable Alzheimer's disease were only included if their MMSE declined over the period of observation of 1-2 years. DTI was performed with a $1.5 \mathrm{~T}$ GE scanner using a single shot, diffusion weighted spin echo EPI sequence. The full details of the acquisition indices have been previously published ${ }^{4}$ but briefly, we used 17 axial oblique slices, TR 10 seconds, TE $105 \mathrm{~ms}$, FOV $30 \mathrm{~cm}$, acquisition 
Table 1 Mean lattice index values for the association and pyramidal WMT fibres in controls and probable Alzheimer's disease cohorts

\begin{tabular}{llll}
\hline & Controls & Grouped AD & Mild ADt \\
\hline Splenium of corpus callosum & $0.65(0.01) \mathrm{l}-\mathrm{r}$ & $0.56(0.02)^{\star \star \star}$ & $0.60(0.02)^{\star}$ \\
& $0.65(0.06) \mathrm{a}-\mathrm{p}$ & $0.43(0.05)^{\star \star \star}$ & $0.46(0.03)^{\star}$ \\
$\quad$ Superior longitudinal fasciculus & $0.46(0.02) \mathrm{a}-\mathrm{p}$ & $0.39(0.01)^{\star \star \star \star}$ & $0.40(0.01)^{\star \star \star \star}$ \\
$\quad$ Cingulum & $0.37(0.01) \mathrm{a}-\mathrm{p}$ & $0.33(0.01)^{\star}$ & $0.35(0.02)$ \\
$\quad$ Left & $0.35(0.01) \mathrm{a}-\mathrm{p}$ & $0.33(0.01)$ & $0.35(0.02)$ \\
$\quad$ Right & $0.39(0.01) \mathrm{v}$ & $0.39(0.01)$ & $0.40(0.01)$ \\
$\quad$ Internal capsule-posterior limb & $0.38(0.01) \mathrm{v}$ & $0.38(0.01)$ & $0.38(0.01)$ \\
$\quad$ Reft arm & & & \\
\hline
\end{tabular}

${ }^{\star} p<0.05,{ }^{\star \star} p<0.01,{ }^{\star \star \star} p<0.005,{ }^{\star \star \star \star \star \star} p<0.0001$.

Measures of anisotropy are given as the lattice index (SEM).

† (MMSE >17); $a-p=$ anterior-posterior; 1 -r=left-right; $v=$ vertically oriented fibres.

matrix $256 \times 128$, reconstructed to $256^{2}, 5 \mathrm{~mm}$ slice thickness, $1 \mathrm{~mm}$ gap per slice, four $\mathrm{b}$ values applied in six diffusion directions $\left(B_{\max }=875 \mathrm{~s} \mathrm{~mm}^{-2}\right)$. The total scanning time was 4 minutes 25 seconds. Brain tissue masks
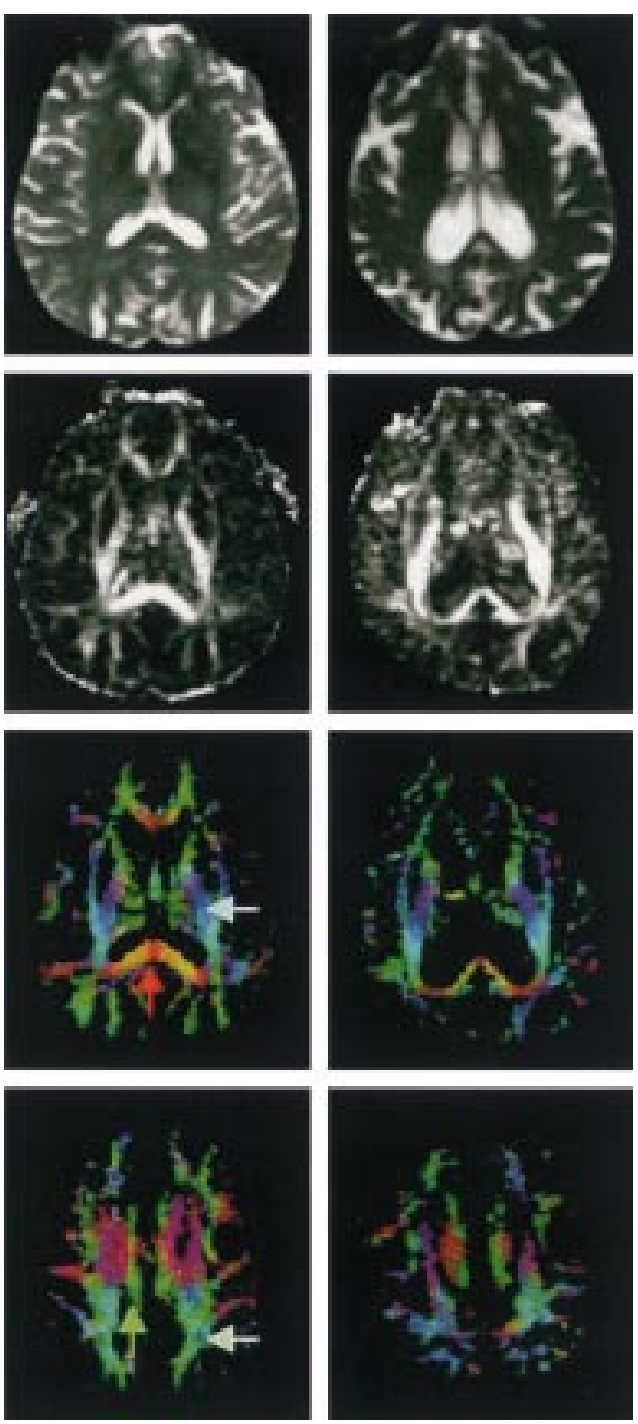

Figure 1 Representative images from an aged matched control (left column) and a patient with probable Alzheimer's disease (right column). The MR images in the rows (top to bottom, respectively) are $T 2$ weighted at the level of the internal capsule, corresponding LI anisotropy images, matching colour coded LI images, and colour coded LI images at the level of the superior longitudinal fasciculus. The splenium (red arrow), superior longitudinal fasciculus (light green arrow), cingulum (dark green), and pyramidal tract (light blue) are demonstrated in the colour coded LI maps. derived from coregistered T2 images (acquired with no diffusion gradients) were used to threshold pixels with anomalous signal from the LI images. Pixels were characterised as either anterior-posterior (green), left-right (red), or vertical (blue) depending on the direction of the eigenvector of the diffusion tensor. ${ }^{3}$ Other colours indicated that the orientation of the fibres was not entirely in a single direction (fig 1). For intrasubject comparison of anisotropy, tracts were clustered into three groups on the basis of whether pixels were oriented within $45^{\circ}$ of each of the three principal directions. Colour coding of the orientation of the fibres allowed ready identification of major WMTs, such as the splenium of the corpus callosum, superior longitudinal fasciculus, cingulum, and vertical fibres of the internal capsule by comparison with previously published data on DTI of white matter association pathways. ${ }^{11}$ For the splenium and cingulum, regions of interest (ROIs) encompassed the entire WMTs. For the vertical fibres of the internal capsule, only the blue colour coded pyramidal tracts were included within the ROI. For the superior longitudinal fasciculus, ROIs were placed in a posterior region of the WMTs encompassing green colour coded anteriorposterior association fibres. Differences between these regions for the two subject groups were analysed by analysis of variance (ANOVA) (table).

\section{Results}

A summary of the measures of the LI for the WMTs in the control and probable Alzheimer's disease cohorts are given in the table. The LI of the splenium of the corpus callosum of patients with probable Alzheimer's disease was highly significantly less than the LI of aged normal controls for left-right oriented fibres and adjoining anteroposteriorly directed callosal fibres. In those patients with mild to moderate probable Alzheimer's disease (MMSE 17-21), the LI was significantly less than that in the normal controls. A similar significant reduction was also seen in the LI of the fibres of the superior longitudinal fasciculus in patients with probable Alzheimer's disease when compared with controls. A significant reduction was also found for the LI of the anterior-posterior fibres of the left cingulum in the probable Alzheimer's disease cohort. No differences in anisotropy were seen between the two groups for the vertically directed WMTs of

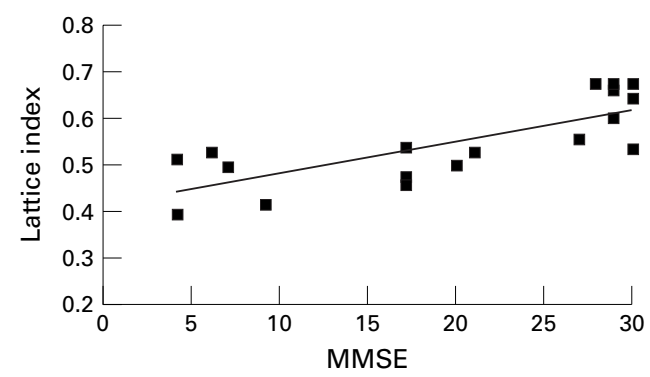

Figure 2 Scatter plot showing the correlation between the LI of the splenium of the corpus callosum and the mini mental state examination score (MMSE). 
the posterior limb of the internal capsule. There was a significant correlation (0.77) between the LI of the splenium of the corpus callosum and the mini mental state examination score (fig 2).

\section{Discussion}

This study shows for the first time significant reductions in the LI of the splenium of the corpus callosum, superior longitudinal fasciculus, and left cingulum in patients with probable Alzheimer's disease. Two previous studies have shown a decrease in the measure of relative anisotropy ${ }^{12} 13$ in the white matter of patients with probable Alzheimer's disease, but neither report correlated anisotropy loss with WMT orientation.

Previous pathological studies of patients with Alzheimer's disease have shown that white matter abnormalities, such as loss of oligodendrocytes and axons, together with reactive astrocytosis are often seen in the parietal lobe white matter and central white matter. ${ }^{1}$ We speculate that such pathological changes in the white matter occurred in the splenium of the corpus callosum, superior longitudinal fasciculus, and left cingulum of patients with probable Alzheimer's disease in this study. Fibres from the splenium and caudal portion of the body of the corpus callosum originate from temporoparietal regions of the brain, ${ }^{14}{ }^{15}$ grey matter regions that are characteristically affected in Alzheimer's disease. ${ }^{1}$ Axon loss in regions of previously high anisotropy will cause a fall in the LI of that region. ${ }^{4}$ It is likely that the loss of callosal connections, due to axonal degeneration, ${ }^{1}$ could account for the fall in the observed LI values. However, reactive astrocytosis and oligodendrocyte loss could also contribute to the fall in LI values in the splenium, superior longitudinal fasciculus, and cingulum of patients with probable Alzheimer's disease. The correlation between the LI of the splenium and the MMSE suggests that a fall in the LI of the splenium of the corpus callosum is related to a decline in cognitive function of these patients.

Further work is required to confirm the proposed link between a loss in the anisotropy of the association fibres of the WMTs and cognitive dysfunction in other dementing illnesses. Our findings serve to highlight the importance of white matter pathology in Alzheimer's disease.

1 Brun A, Englund E. A white matter disorder in dementia of the Alzheimer type. A pathoanatomical study. Ann Neurol 1986;19:253-62.

2 Pierpaoli C, Jezzard P, Basser PJ, et al. Diffusion tensor MR imaging of the human brain. Radiology 1996;201:637-48.

3 Pajevic S, Pierpaoli C. Colour schemes to represent the orientation of anisotropic tissues from diffusion tensor data: entation of anisotropic tissues from diffusion tensor data: application to white mattter fiber tract mapping
human brain. Magn Reson Med 1999;42:526-40.

4 Zelaya F, Flood N, Chalk JB, et al. An evaluation of the time dependence of the anisotropy of the water diffusion tensor in acute human ischemia. Magn Reson Imaging 1999;17: 331-48.

5 Werring DJ, Clark CA, Barker GJ, et al. The structural and unctional mechanisms of motor recovery: complementary use of diffusion tensor and functional magnetic resonance imaging in a traumatic injury of the internal capsule. 7 Neurol Neurosurg Psychiatry 1998;65:863-9.

6 Goldman WP, Baty JD, Buckles VD, et al. Motor dysfunction in mildly demented $\mathrm{AD}$ individuals without extrapyramidal signs. Neurology 1999;53:956-62.

7 Neary D, Snowden JS, Gustafson L, et al. Frontotemporal lobar degeneration: a consensus on clinical diagnostic lobar degeneration: a consensus on

8 Folstein MF, Folstein SF, McHugh PR. Mini mental state: a practical method for grading the cognitive state of patients for the clinician. F Psychiatr Res 1975;12:189-98.

9 McKhann G, Drachman D, Folstein M, et al. Clinical diagnosis of Alzheimer's disease: report of the NINCDSADRDA Work Group under the auspices of Department of Health and Human Services Task Force on Alzheimer's Disease. Neurology 1984;34:939-44.

10 American Psychiatric Association. Diagnostic and statistical manual of mental disorders. 3rd ed, revised. Washington, DC: American Psychiatric Association, 1987:101-28.

11 Makris N, Worth AJ, Sorensen AG, et al. Morphometry of in vitro human white matter association pathways with diffusion-weighted magnetic resonance imaging. Ann Neurol 1997;42:951-62.

12 Haruo $\mathrm{H}$, Hiroaki S, Dai K, et al. Increased water diffusion in cerebral white matter in Alzheimer's disease. Gerontology 1997;43:343-51.

13 Sandson T, Felician O, Edelman P, et al. Diffusion-weighted magnetic resonance imaging in Alzheimer's disease. Dement Geriatr Cogn Disord 1999;10:166-71

14 de Lacoste MC, Kirkpatrick JB, Ross ED. Topography of the human corpus callosum. I Neuropathol Exp Neurol $1985 ; 44: 578-91$.

15 Conturo TE, Lori NF, Cull TS, et al. Tracking neuronal fiber pathways in the living human brain. Proc Natl Accad Sci USA 1999;96:10422-7. 